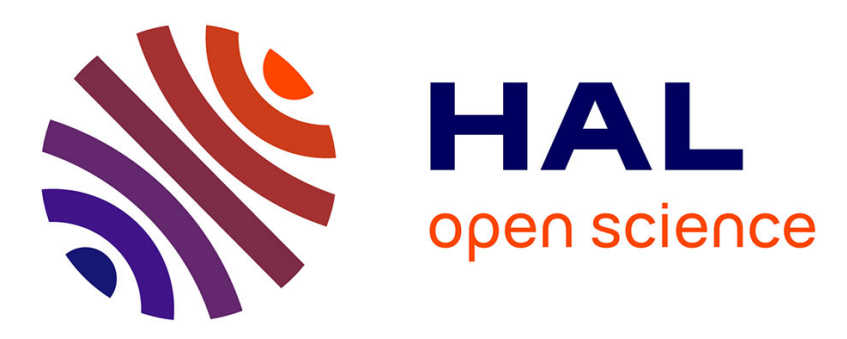

\title{
Crossed ratchet effects on magnetic domain walls: geometry and transverse field effects
}

\author{
A Alija, A Hierro-Rodríguez, A Pérez-Junquera, Jm Alameda, Ji Martín, M \\ Vélez
}

\section{- To cite this version:}

A Alija, A Hierro-Rodríguez, A Pérez-Junquera, Jm Alameda, Ji Martín, et al.. Crossed ratchet effects on magnetic domain walls: geometry and transverse field effects. Journal of Physics D: Applied Physics, 2011, 44 (32), pp.325002. 10.1088/0022-3727/44/32/325002 . hal-00642371

\section{HAL Id: hal-00642371 https://hal.science/hal-00642371}

Submitted on 18 Nov 2011

HAL is a multi-disciplinary open access archive for the deposit and dissemination of scientific research documents, whether they are published or not. The documents may come from teaching and research institutions in France or abroad, or from public or private research centers.
L'archive ouverte pluridisciplinaire HAL, est destinée au dépôt et à la diffusion de documents scientifiques de niveau recherche, publiés ou non, émanant des établissements d'enseignement et de recherche français ou étrangers, des laboratoires publics ou privés. 


\title{
Crossed ratchet effects on magnetic domain walls: geometry and transverse field effects
}

\author{
A Alija, A Hierro-Rodríguez, A Pérez-Junquera, JM Alameda, JI Martín and M \\ Vélez \\ Dept. Física, Universidad de Oviedo-CINN, 33007 Oviedo, SPAIN \\ E-mail: $\underline{\text { mvelez@uniovi.es }}$
}

\begin{abstract}
Domain wall propagation across a 2D array of asymmetric holes is strongly dependent on domain wall configuration: i.e. on whether the wall is flat or kinked. This results in interesting crossed ratchet and asymmetric accommodation effects that have been studied as a function of geometry and transverse field. Micromagnetic simulations have shown that the observation of crossed ratchet effects is easier for arrow than for triangular holes due to a larger field range in which kink propagation is the preferred mode for domain wall motion. Also, it has been found that dc transverse fields can produce a significant enhancement of the easy axis asymmetric accommodation and, also, that ac transverse fields can be rectified by the crossed ratchet potential.
\end{abstract}

PACS: 75.60Ch, 75.60.Jk; 75.75.-c

Short title: Crossed ratchet effects on domain walls: geometry and transverse field effects 


\section{Introduction}

A good understanding of domain wall (DW) propagation in magnetic nanostructures is a key issue for device applications [1,2]. A case of particular interest is the study of ratchet effects that create an asymmetry between forward/backward domain wall propagation. This asymmetry can be of use for the design of DW diodes [3-8] and memory devices [9]. A DW ratchet appears whenever the relevant pinning potential is asymmetric either due to an asymmetric geometry as in magnetic nanowires of triangular section [3] or an asymmetric domain wall configuration as in Neel walls of different chirality [10]. Two different sorts of DW ratchets can be found depending on the nanostructure dimensionality: simple forward/backward ratchet in patterned 1D nanowires [3-8,10] and crossed ratchets in 2D arrays of asymmetric pinning centers $[9,11]$. In the first case the sign of the ratchet asymmetry is fixed by nanostructure geometry and/or DW chirality whereas in the second case the sign of the ratchet asymmetry depends on the applied field amplitude and DW configuration. Thus, crossed ratchets allow for a more complex control of DW propagation that can be tuned both by array geometry and external parameters.

Up to now crossed ratchet effects have only been experimentally demonstrated in arrays of arrow holes [9], whereas most of the theoretical analysis and simulations of this phenomenon have been performed using the simpler triangular geometry [11,12]. Also, the pinning interaction between a Neel wall and an array of symmetric patterned holes is known to be quite sensitive to changes in domain wall energy [13] that could be tuned by the application of a small transverse field. In this work we have combined micromagnetic simulations and magnetization measurements in order to analyze the role of two different factors on crossed ratchet effects created by an array of asymmetric holes: hole shape (comparing triangular and arrow holes) and the effect of transverse fields applied along the hard anisotropy axis.

\section{Experimental and simulation details}

$500 \mu \mathrm{m} \times 500 \mu \mathrm{m}$ arrays of asymmetric holes arranged in a $20 \mu \mathrm{m} \times 20 \mu \mathrm{m}$ square lattice have been fabricated in $40 \mathrm{~nm}$ thick amorphous $\mathrm{Co}_{73} \mathrm{Si}_{27}$ films by a combined e-beam lithography and etching process, as reported elsewhere [14]. The magnetic films show a well defined in-plane uniaxial anisotropy $\left(K=1000 \mathrm{~J} / \mathrm{m}^{3}\right)$, low saturation magnetization $\left(M_{\mathrm{S}}=\right.$ $2 \times 10^{5} \mathrm{~A} / \mathrm{m}$ ) and low coercivity [13]. The asymmetric holes have an arrow shape pointing in a direction perpendicular to the easy axis, as shown in Fig. 1. The arrow shape defines the "forward" (i.e. left to right) propagation sense (correspondingly "backward" propagation indicates right to left DW motion). The magnetic behavior of the patterned films was characterized by Magnetooptical Transverse Kerr (MOTKE) effect, using a set up in which the laser beam is focused in a $300 \mu \mathrm{m}$ spot, i.e. smaller than the patterned area [15]. The magnetic field $H$ was always applied in the sample plane. 


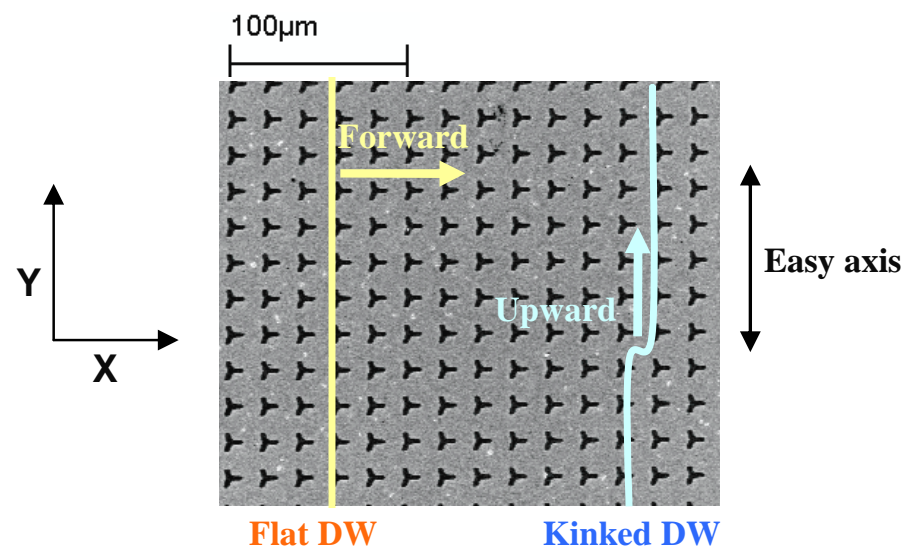

Figure 1 (colour online) SEM micrograph of a square array of arrow holes. Solid lines are sketches of a flat and a kinked DW. Arrows indicates forward DW propagation sense and upward kink propagation sense. Note that Upward kink propagation is equivalent to net backward DW motion.

Micromagnetic simulations of DW propagation across similar patterned arrays have been performed with the OOMMF code [16]. Square arrays of empty triangular/arrow holes have been defined in rectangular thin film elements with material parameters corresponding approximately with those of the Co-Si alloy: $M_{\mathrm{S}}=2 \times 10^{5} \mathrm{~A} / \mathrm{m}, A=3 \times 10^{-11} \mathrm{~J} / \mathrm{m}$ and uniaxial anisotropy $K=1000 \mathrm{~J} / \mathrm{m}^{3}$ with the easy axis oriented in the $y$ direction. Typical mesh sizes are $15-35 \mathrm{~nm}$, smaller than both the material exchange length, $\delta_{\mathrm{ex}}=\left(2 A / \mu_{0} M_{\mathrm{S}}^{2}\right)^{1 / 2}=35 \mathrm{~nm}$, and the Bloch parameter $\delta_{0}=(A / K)^{1 / 2}=170 \mathrm{~nm}$.

\section{Asymmetric pinning potential and hole geometry: micromagnetic simulations}

DW propagation across a 2D array of asymmetric defects is characterized by two different field scales corresponding to the softer propagation mode in each sense [9,11]: $H_{F}$ the field needed for forward flat wall propagation and $H_{U}$ the field needed for upward kink propagation in kinked walls which is equivalent to net backward wall motion (see sketches in Fig.1). In the field range between $H_{F}$ and $H_{U}$ the system displays the typical signatures of crossed ratchet behaviour [9]: inverted asymmetry of minor hysteresis loops relative to major loops and asymmetric accommodation curves that keep memory of the sign of the last saturating state. In this section we will study domain wall propagation across identical arrays of $4 \mu \mathrm{m}$ holes changing only hole shape (triangular vs. arrow) both for flat and kinked wall configurations.

\subsection{Simulated magnetization curves and energy landscapes}

Figure 2 shows the calculated magnetization curves as a flat DW crosses the sample for a square array of $4 \mu \mathrm{m}$ triangular holes (Fig. 2(a)) and for a square array of $4 \mu \mathrm{m}$ arrow holes (Fig. 2(b)). The simulations start from an initial zero field-zero magnetization state with a straight $180^{\circ}$ Neel wall located at the film center (see frames I in Fig.2). 

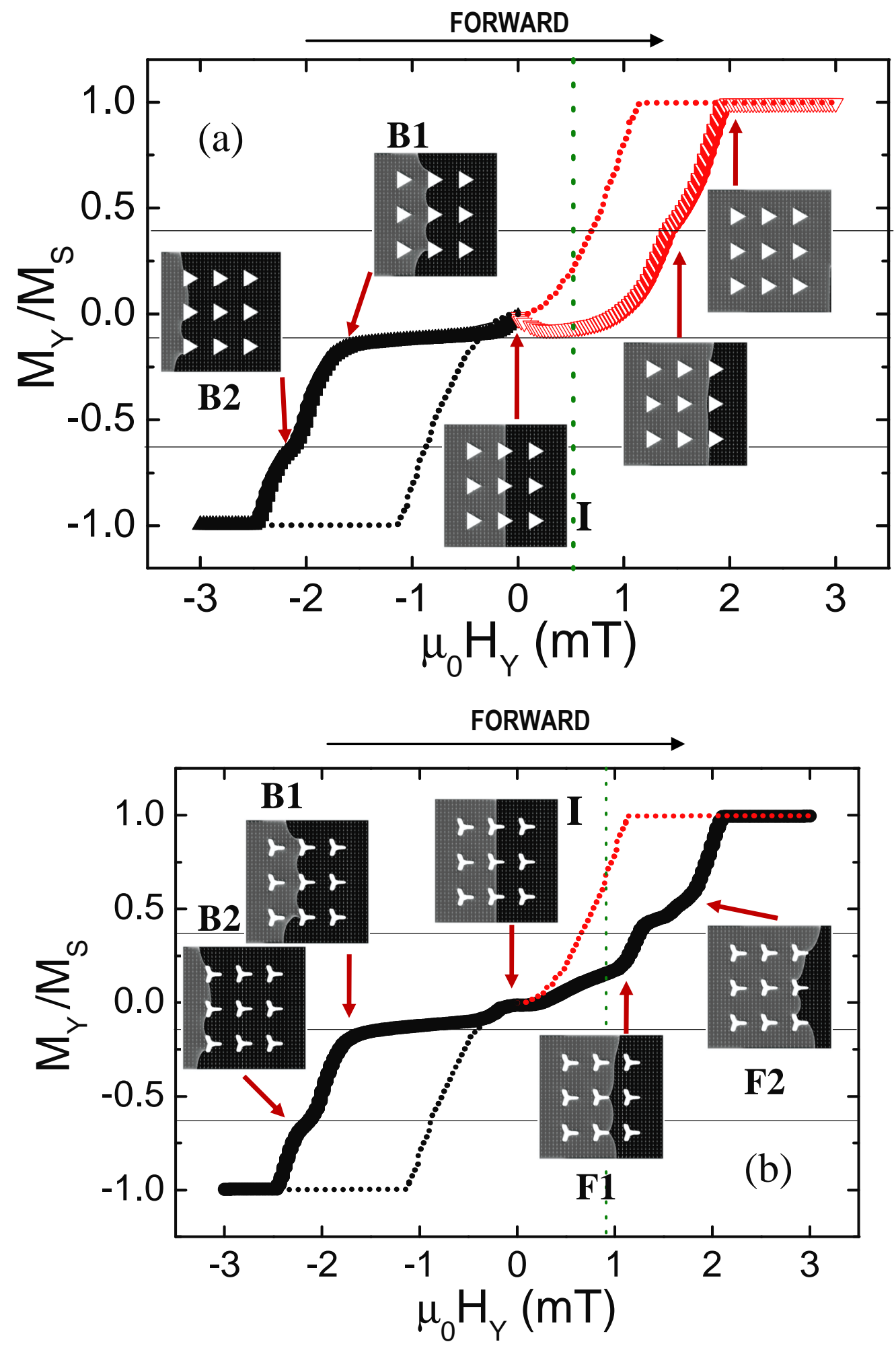

Figure 2 (colour online) Calculated $\mathrm{M}_{\mathrm{Y}} v s \mathrm{H}_{\mathrm{Y}}$ for a CoSi film starting from an initial magnetization state with a $180^{\circ} \mathrm{Neel}$ wall at the film center, when the wall is either pushed forward or backward (positive/negative $H$ ) with (a) an array of $3 \times 3$ triangles $(4 \mu \mathrm{m})$ and (b) an array of $3 \times 3$ arrows (4 $\mu \mathrm{m})$. Dotted line indicates the calculated response of a similar continuous CoSi square. Insets show the wall configuration at selected positions in the curve. (colour code: black = negative $\mathrm{M}_{\mathrm{Y}}$; grey = positive $\mathrm{M}_{\mathrm{Y}}$ ). Horizontal straight lines indicate $M_{\mathrm{y}} / M_{\mathrm{S}}$ corresponding to a flat wall pinned at each of the defect lines and vertical straight lines indicates $H_{\mathrm{F}}$ as derived from energy landscapes. 
Then, this wall is pushed either forward or backward by a continuous positive/negative field $H_{\mathrm{y}}$ of increasing amplitude applied along the easy axis for uniaxial anisotropy until positive/negative saturation is reached. The magnetization curves develop a series of steps that correspond approximately with the $M_{\mathrm{y}} / M_{S}$ values expected for a DW being pinned at each of the three lines of holes that form the patterned arrays (indicated as solid horizontal lines in Fig. 2). Also, the presence of the array of holes results in a significant enhancement of the saturation field $H_{S}$ in comparison with the behavior of an unpatterned Co-Si element (shown as a dotted line for comparison). In both cases (arrows and triangles), $H_{S}$ is clearly larger for backward than for forward propagation as expected from the individual hole asymmetry. It is interesting to note that backward magnetization curves are very similar for both kinds holes, which can be attributed to the similarities in the relevant backward DW propagation mode: DW depinning starts from the DW segment located between the lower film border and the last hole that, after reaching a critical curvature radius, slides along the vertical hole side (see frames B1 and B2 in Fig. 2). However, the differences in hole shape clearly show up in forward propagation curves that present broader steps for triangles than for arrows and a larger saturation field in the second case.

Further information on the DW propagation process can be obtained by energy landscapes calculated as the wall crosses the array of defects under a constant applied field, [12] that allow us to separate the different possible DW propagation modes in a more accurate way than with the global magnetization curves. Figure 3(a) shows the difference between total energy $E_{T}$ and Zeeman energy $E_{Z}$ as a function of domain wall position (given approximately by $\left.M_{\mathrm{y}} / M_{\mathrm{S}}\right)$ as a flat DW crosses a line of $4 \mu \mathrm{m}$ arrow holes. The energy curve has the typical triangular shape of ratchet potentials with a much lower slope in the forward than in the backward propagation sense, i.e. forward is the easiest propagation sense. A similar calculation starting from an initial kinked wall pinned in between two lines of arrows can be seen in Fig. 3(b). The energy landscape displays again a triangular potential well but with opposite asymmetry than for the flat wall depicted in Fig. 3(a): now the gradual slope corresponds to net backward DW motion (i.e. as the kink proceeds upward). This change in potential asymmetry depending on the geometrical DW configuration is the essential ingredient of crossed ratchet behavior and can only be observed in 2D systems.

\subsection{Analysis of characteristic fields}

In order to make a more quantitative analysis of the asymmetric domain wall propagation several characteristic fields can be defined from the calculated curves of Figs. 2 and 3 as indicated in Table 1. First, the global effect of the array of holes on DW propagation can be characterized by the hardening of the magnetization curve: 

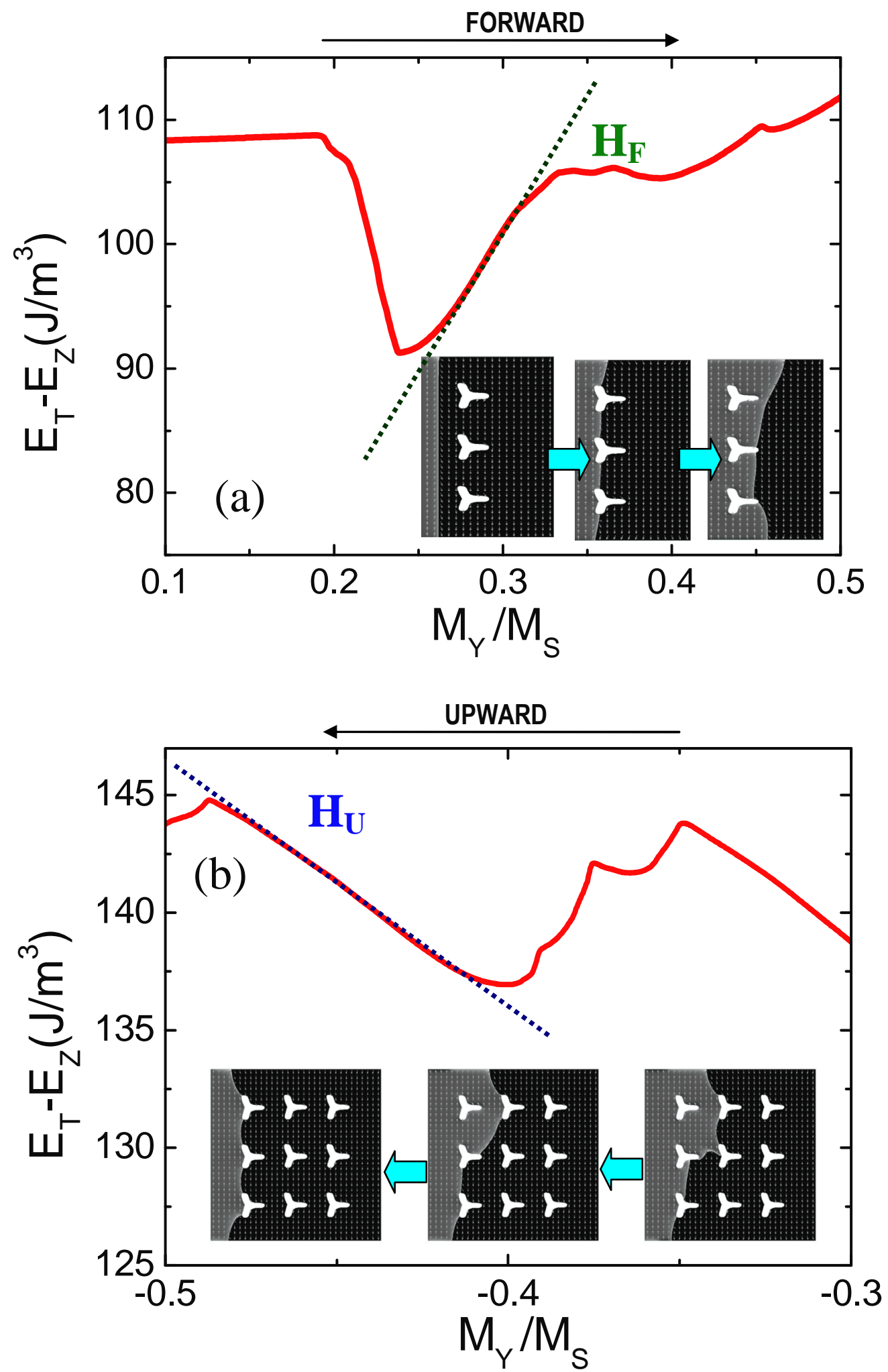

Figure 3 (colour online) (a) Simulated energy landscape as a function of $M_{y} / M_{S}$ as a flat wall crosses a line of $4 \mu \mathrm{m}$ arrow holes propagating forward under a constant applied field of $3 \mathrm{mT}$. (b) Simulated energy landscape as a function of $\mathrm{M}_{\mathrm{y}} / \mathrm{M}_{\mathrm{S}}$ as a kink proceeds upward along a wall pinned in between two lines of $4 \mu \mathrm{m}$ arrow holes under a constant applied field of $-3 \mathrm{mT}$. Frames correspond to selected wall configurations along the DW propagation process. 


$$
\Delta \mathrm{H}_{\mathrm{F} / \mathrm{B}}=H_{S}(\text { Forward/Backward })-H_{S}(\text { unpatterned }),
$$

For backward propagation, the hardening induced by the arrays of holes is the same for arrows and triangles: $\mu_{0} \Delta \mathrm{H}_{\mathrm{B}}($ arrow $)=\mu_{0} \Delta \mathrm{H}_{\mathrm{B}}($ triangle $)=1.3 \mathrm{mT}$, and the differences in shape show up in forward depinning: $\mu_{0} \Delta \mathrm{H}_{\mathrm{F}}$ (arrow) $=1 \mathrm{mT}$ and $\mu_{0} \Delta \mathrm{H}_{\mathrm{F}}$ (triangle) $=0.8 \mathrm{mT}$. These results can be understood using as a first approximation a simplified analytical model with only DW elastic energy and applied field pressure terms [11]. In this model, the critical field for propagation of a DW pinned in between two surfaces of arbitrary shape (such as in between two holes or in between a hole and a border) is inversely proportional to inter hole distance (or hole-border distance) and depends also on the angles made by the limiting surfaces: for triangle holes, the analytical model predicts $\mathrm{H}_{\mathrm{F}} / \mathrm{H}_{\mathrm{B}}=\sin \left(30^{\circ}\right)=0.5$ roughly in agreement with the data in Table $1, \Delta \mathrm{H}_{\mathrm{F}} / \Delta \mathrm{H}_{\mathrm{B}}=0.61$; on the other hand, for arrow holes, the results extracted from the simulated magnetization curves give $\Delta \mathrm{H}_{\mathrm{F}} / \Delta \mathrm{H}_{\mathrm{B}}=0.77$ which lies in between the asymmetry expected for depinning from the arrow base $\mathrm{H}_{\mathrm{F}} / \mathrm{H}_{\mathrm{B}}=\sin \left(68^{\circ}\right)=0.93$ and from the arrow tip $\mathrm{H}_{\mathrm{F}} / \mathrm{H}_{\mathrm{B}}=4 \mu \mathrm{m} / 6 \mu \mathrm{m}=0.67$ (see frames $\mathrm{F} 1$ and $\mathrm{F} 2$ in Fig.2(b)).

Table 1: Characteristic fields of the arrays of $4 \mu \mathrm{m}$ arrow and triangular holes.

\begin{tabular}{|lcccccc|}
\hline & $\begin{array}{c}\mu_{0} \Delta \mathbf{H}_{\mathrm{F}} \\
(\mathrm{mT})\end{array}$ & $\begin{array}{c}\mu_{0} \Delta \mathbf{H}_{\mathbf{B}} \\
(\mathrm{mT})\end{array}$ & $\begin{array}{c}\mu_{0} \mathbf{H}_{\mathrm{F}} \\
(\mathrm{mT})\end{array}$ & $\begin{array}{c}\mu_{0} \mathbf{H}_{\mathbf{U}} \\
(\mathrm{mT})\end{array}$ & $\begin{array}{c}\mu_{0} \Delta \mathbf{H}_{\text {Cross }}=\mu_{0} \mathbf{H}_{\mathrm{F}}-\mu_{0} \mathbf{H}_{\mathbf{U}} \\
(\mathrm{mT})\end{array}$ \\
\hline Triangles & 0.8 & 1.3 & 0.5 & 0.3 & 0.2 \\
\hline Arrows & 1 & 1.3 & 0.9 & 0.5 & 0.4 \\
\hline
\end{tabular}

However, it must be noted that in the global characterization of the asymmetric behavior obtained from the equilibrium magnetization curves, different factors contribute to DW propagation (depinning from film borders, depinning from inter-hole regions, demagnetizing effects of the square Co-Si element...). This results in relatively broad transitions between DW pinning states at consecutive defect lines that make difficult a sharp definition of the critical field. Better defined depinning fields can be obtained for each particular DW propagation mode from the slopes of the triangular wells that appear in the energy landscapes shown in Fig.3. For flat walls, the results are $\mu_{0} H_{\mathrm{F}}$ (arrows) $=0.9 \mathrm{mT}$ and $\mu_{0} H_{\mathrm{F}}$ (triangles) $=0.5 \mathrm{mT}$ (dotted vertical lines have been plotted in Fig. 2 at these field positions and correspond roughly with the start of the DW transition from the central to the rightmost line of defects). For kink propagation, the upward propagation field $H_{\mathrm{U}}$ estimated from the gradual slope is $\mu_{0} H_{\mathrm{U}}=0.3 \mathrm{mT}$ for triangles and $0.5 \mathrm{mT}$ for arrow holes, which are clearly lower values than the corresponding $\mu_{0} H_{\mathrm{F}}$.

The interval between $\mu_{0} H_{\mathrm{U}}$ (the critical field for the softer backward propagation mode) and $\mu_{0} H_{\mathrm{F}}$ (the critical field for the softer forward propagation mode) is, then, the available range 
for observation of crossed ratchet effects dominated by kink motion. The calculated values are $\mu_{0} \Delta \mathrm{H}_{\text {Cross }}($ arrow $)=\mu_{0} \mathrm{H}_{\mathrm{F}}-\mu_{0} \mathrm{H}_{\mathrm{U}}=0.4 \mathrm{mT}$ and $\mu_{0} \Delta \mathrm{H}_{\text {Cross }}$ (triangle $)=0.2 \mathrm{mT}$, so that for a given array geometry the observation of crossed ratchet effects should be easier of arrays of arrow holes. In order to make a qualitative comparison of this calculation in square arrays of $4 \mu \mathrm{m}$ arrow holes with experimental results in square arrays of $10 \mu \mathrm{m}$ arrow holes, size effects can be considered by applying the proper scale factor between global array dimensions 4/10 [11,12]. Thus, the scaled range becomes $\mu_{0} \Delta \mathrm{H}_{\text {Cross }}{ }^{\text {CALC }}=0.16 \mathrm{mT}$ in quite a good agreement with the experimental $\mu_{0} \Delta \mathrm{H}_{\text {Cross }}{ }^{\mathrm{EXP}}=0.2 \mathrm{mT}[9]$.

\section{Rectification enhancement in a transverse field}

Neel walls, with their complex tail-core structure, are known to be quite sensitive to hard axis magnetic fields [17-19]. Thus, transverse magnetic fields could be of use to tune DW propagation across the array of asymmetric holes. In particular, one of the most interesting features in a crossed ratchet is the so called asymmetric accommodation [9]. This phenomenon appears when a DW is introduced in the array of asymmetric holes and, then, the system is excited by a small ac field. As shown previously [9], the walls enter flat into the array and develop kinks as they move forward due to small pinning center inhomogeneities and/or Barkhausen jumps. The ac field allows the pinned wall to relax to a lower energy state in a process governed by kink propagation under the asymmetric crossed ratchet potential.

\subsection{Transverse field experiments}

The influence of a transverse field on asymmetric accommodation can be clearly seen in Figure 4 in which the results of series of asymmetric accommodation experiments performed under different values of a constant transverse field $H_{\mathrm{x}}$ are shown: Fig. 4(a) displays the easy axis applied field sequence $H_{\mathrm{y}}(t)$ and Fig. 4(b) the measured easy axis magnetization response $M_{\mathrm{y}}(\mathrm{t})$. The experiment begins when a large negative field $H_{\mathrm{y}}$ is applied to the array of holes at a time $t=0.06 \mathrm{~s}$ in order to saturate the array in the negative direction. Then, $H_{\mathrm{y}}$ is increased up to the positive coercivity in order to introduce a DW in the array and, finally, it is reduced to zero at $t=0.11 \mathrm{~s}$, in order to prepare a remanent state with a small magnetization indicative of a DW pinned inside the array. Now, after $t=0.16 \mathrm{~s}$, a small oscillating field of increasing amplitude $H_{\text {y_ac }}$ is applied to the array so that the pinned wall is alternatively pushed forward and backward. For very low $H_{y_{-} \text {ac }}$ the wall stays pinned but, when $H_{y_{\_} \text {ac }}$ is within $\Delta \mathrm{H}_{\text {Cross }}$ (i.e. $H_{\mathrm{U}}<$ $H_{\text {y_ac }}<H_{\mathrm{F}}$ ) only kink propagation is allowed and the DW displays a net backward motion that results in the asymmetric accommodation of the magnetization towards its last saturating value ( $-M_{\mathrm{S}}$ in this case): that is, the ac field excitation produces a rectified backward DW propagation that gives rise to a constant magnetization change. This process takes place approximately 

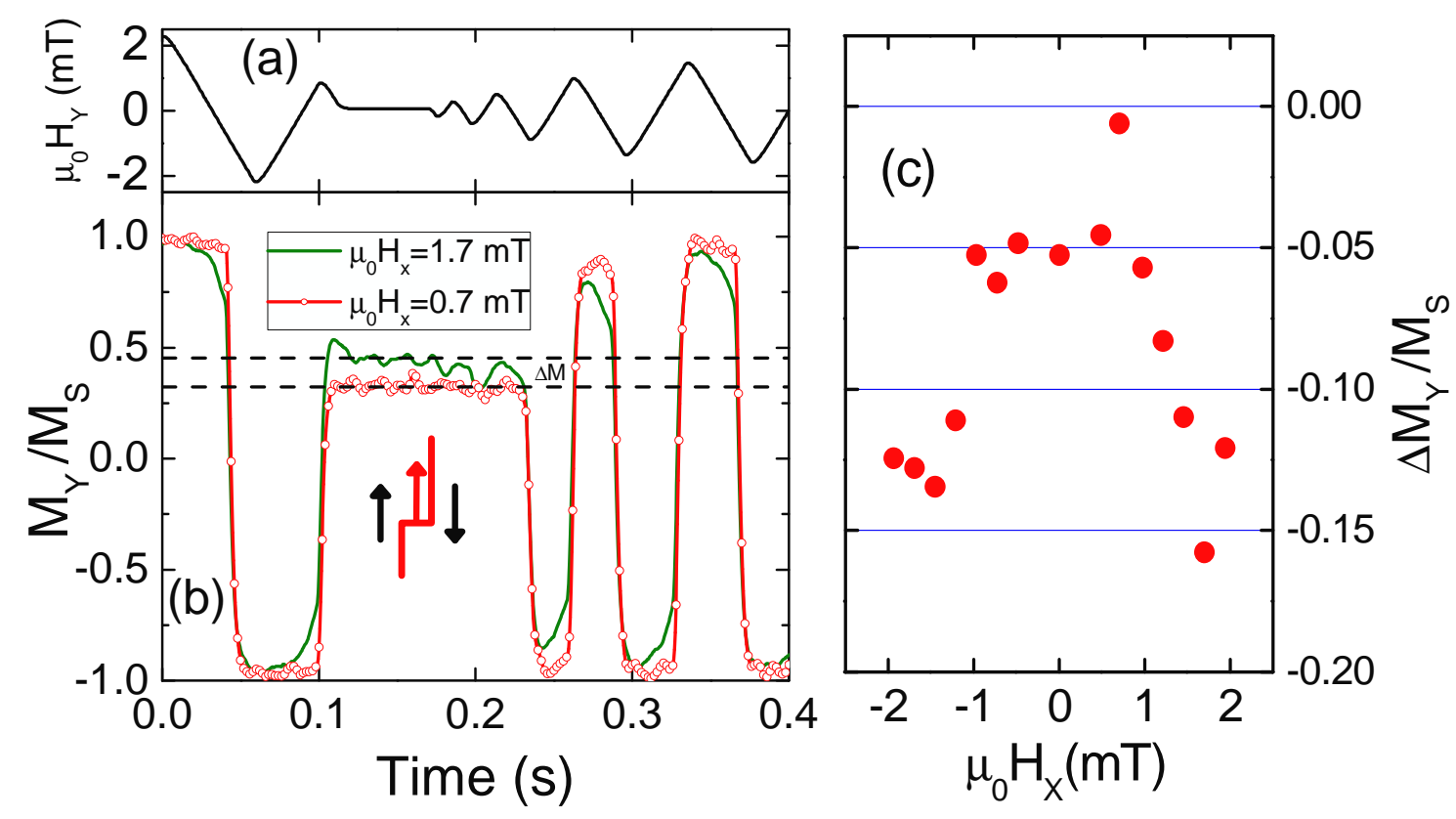

Figure 4 (colour online) Asymmetric accommodation under different values of a DC transverse field: (a) Easy axis $\mu_{0} \mathrm{H}_{\mathrm{Y}}(\mathrm{t})$ applied field sequence (b) measured easy axis $\mathrm{M}_{\mathrm{Y}}(\mathrm{t})$ response. Dashed horizontal lines indicate asymmetric accommodation $\Delta \mathrm{M}$ under $\mu_{0} \mathrm{H}_{\mathrm{X}}=1.7 \mathrm{mT}$. (c) Asymmetric accommodation $\Delta \mathrm{M}_{\mathrm{Y}} / \mathrm{M}_{\mathrm{S}}$ as a function of transverse field $\mu_{0} \mathrm{H}_{\mathrm{X}}$.

within $0.16 \mathrm{~s}<t<0.23 \mathrm{~s}$ and can be characterized by the small negative $\Delta M_{\mathrm{y}}$ that occurs during this time interval (see dashed horizontal lines in Fig. 4(b)). Eventually, $H_{\text {y_ac }}$ becomes large enough to allow extra DWs to be nucleated at the array limits (i.e. $H_{\mathrm{F}}<H_{\mathrm{y}_{\mathrm{a}} \mathrm{ac}}$ ) and large positive/negative magnetization oscillations are observed in the $M(t)$ curve due to the growth and annihilation of domains within the patterned array. The accommodation $\Delta M_{\mathrm{y}}$ is quite different for the two curves plotted in Fig. 4(b), with a much higher value for the curve recorded with the larger transverse field $\mu_{0} H_{\mathrm{x}}=1.7 \mathrm{mT}$. Figure $4(\mathrm{c})$ shows the net magnetization increments during the accommodation process as a function of the transverse applied field. $\Delta M_{\mathrm{y}}$ is almost constant (about $0.05 M_{\mathrm{S}}$ ) at low fields but is enhanced more than twofold up to $0.12 M_{\mathrm{S}}$ for $\mu_{0} H_{\mathrm{x}}$ above $1 \mathrm{mT}$.

Figure 5 shows the results of a different transverse field experiment: in this case, the system is first taken to its negative saturation by a large negative easy axis field, then $H_{\mathrm{y}}$ is increased close to the positive coercivity in order to introduce a DW in the patterned area and, finally, it is reduced to zero so that a DW is left pinned at remanence inside the array of holes. Now a transverse ac field $H_{\mathrm{ac}-\mathrm{x}}$ of fixed amplitude is applied to the sample. The corresponding $M_{\mathrm{y}}(\mathrm{t})$ signal shows a continuous decrease towards $-M_{\mathrm{S}}$ modulated by a small ripple at a double frequency than the excitation field. This ripple is just a signature of the uniaxial film transverse susceptibility [20]: the hard axis magnetization component is given by $M_{\mathrm{x}} / M_{\mathrm{S}}=H_{\mathrm{x}} / H_{\mathrm{K}}$ and, 


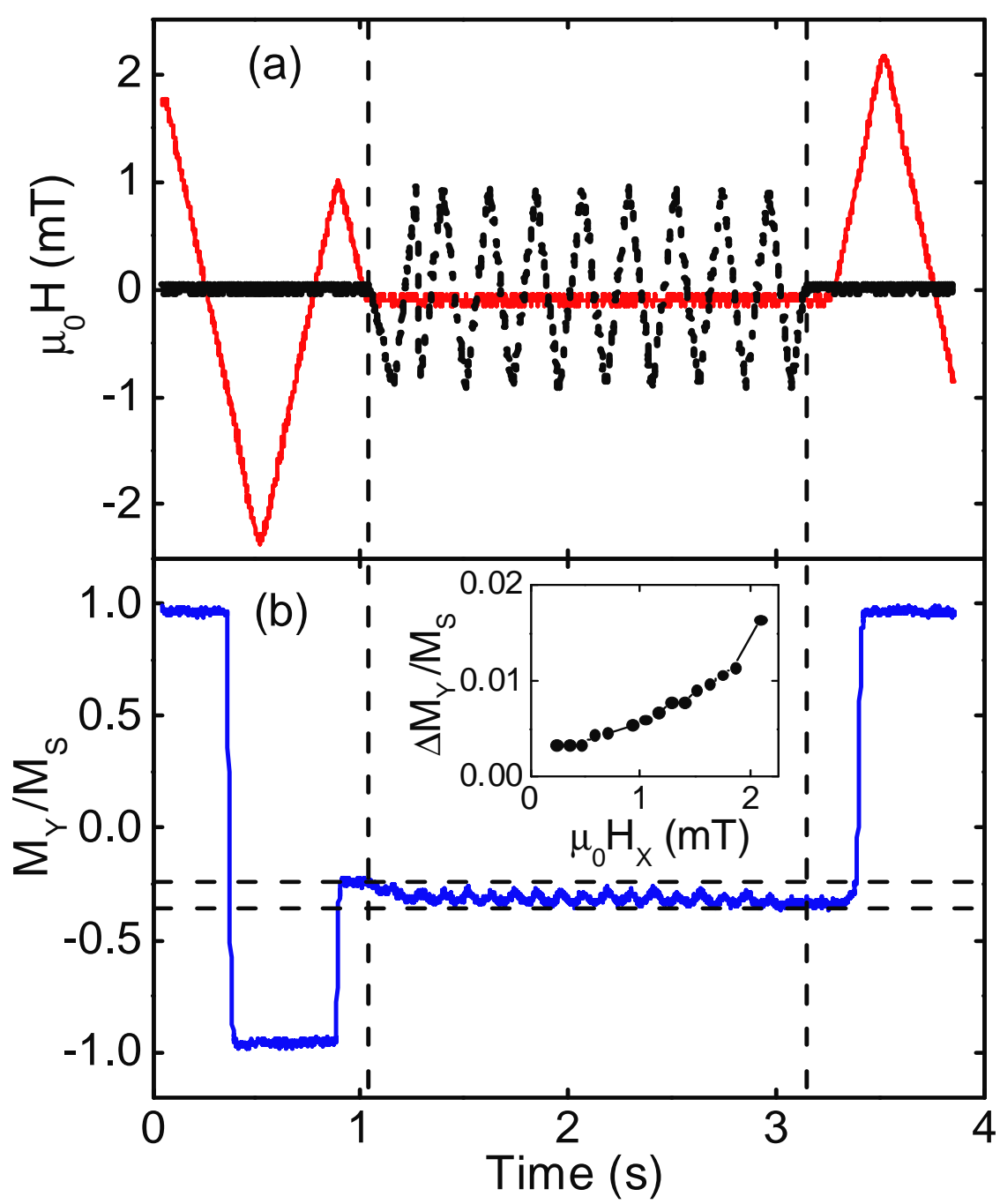

Figure 5 (colour online) (a) Applied field sequence in order to introduce a DW in the array and probe its response to an AC transverse field: $\mu_{0} \mathrm{H}_{\mathrm{Y}}(\mathrm{t})$, solid line; $\mu_{0} \mathrm{H}_{\mathrm{X}}(\mathrm{t})$, dashed line; (b) Measured time dependence of the magnetization under an $\mathrm{AC}$ transverse field. Dashed lines indicate the time interval for $\mathrm{AC}$ transverse field application and the induced asymmetric accommodation $\Delta \mathrm{M}_{\mathrm{Y}} / \mathrm{M}_{\mathrm{S}}$. Inset shows $\Delta \mathrm{M}_{\mathrm{Y}} / \mathrm{M}_{\mathrm{S}}$ as a function of the amplitude of the transverse AC field.

correspondingly, $M_{\mathrm{y}} / M_{\mathrm{S}}=\left[1-\left(M_{\mathrm{x}} / M_{\mathrm{S}}\right)^{2}\right]^{1 / 2} \approx 1-1 / 2\left(H_{\mathrm{x}} / H_{\mathrm{K}}\right)^{2}$. This quadratic dependence of $M_{\mathrm{y}}$ vs. $H_{\mathrm{x}}$ is responsible of the frequency doubling of the observed ripple. More interesting is the observed net magnetization decrease $\Delta M_{\mathrm{y}}$ produced by the ac hard axis field $H_{\mathrm{ac} \_\mathrm{x}}$ : it is of the same sign as in the easy axis experiments of Fig.4 and takes very similar values (in the $0.1 M_{\mathrm{S}}$ $0.2 M_{\mathrm{S}}$ range as shown in the inset of Fig. 5). These results suggest that the transverse field excitation is rectified by the crossed ratchet potential by a similar mechanism as in the easy axis case: i.e. by the net backward motion of the pinned wall due to low field kink propagation.

\subsection{Discussion on transverse field effects}

The experimental results presented in Figs. 4 and 5 show a two fold effect of a transverse field on domain wall propagation inside the asymmetric array of holes: asymmetric 
accommodation is enhanced by the presence of a transverse dc field and, also, asymmetric accommodation can be produced by the application of a transverse ac field of small amplitude. This is quite interesting from the point of view of applications, since memory devices based on crossed ratchets rely on the observation of asymmetric accommodation [9] and transverse fields could be of use for sensitivity optimization.

In order to better understand the results presented above, we have performed a series of micromagnetic simulations to analyze the influence of a transverse field on a pinned kinked DW (see Fig.6): we start from an initial zero field configuration of a kinked DW pinned in between two lines of arrows, then a positive/negative transverse field of constant amplitude is applied to the patterned film and, finally, $H_{\mathrm{x}}$ is reduced again to zero and the system is allowed to relax to a new magnetization configuration (see $\mu_{0} H_{\mathrm{x}}(\mathrm{t})$ and $M_{\mathrm{y}}(\mathrm{t})$ in Figs. 6(a) and (b), respectively). Snapshots of the resulting magnetization configuration are shown at different moments of the simulations labeled as I (initial), II (relaxed under a constant transverse field) and III (final configuration after removing the transverse field) for a positive transverse field pulse (Fig. 6(c)) and for a negative transverse field pulse (Fig. 6(d)). Initially the system starts from $M_{\mathrm{y}}=-0.4 M_{\mathrm{S}}$ and a wall with a diagonal kink crossing the upper-left cell of the array of arrows. Then, upon application of the transverse field, there is a global rotation of the magnetic moments towards the applied field direction that produces a reduction of the absolute value of the easy axis magnetization down to $M_{\mathrm{y}}=-0.1 M_{\mathrm{S}}$ very similar both for positive/negative transverse fields (see frames II). This rotation is particularly intense in the shaded areas just below/above the arrow holes but it is reversible (i.e. it disappears as soon as the applied field is reduced to zero). It corresponds to the transverse susceptibility effect that showed up as the double frequency ripple in the experiments. On the other hand, there is an irreversible component in the simulated magnetization process that depends clearly on the sign of the transverse field: for $\mu_{0} H_{\mathrm{x}}=8 \mathrm{mT}$ the kink has dissolved and there is only a very broad flat DW pinned at the first line of defects (see frame II in Fig. 6(c)), whereas for $\mu_{0} H_{\mathrm{x}}=-8 \mathrm{mT}$ the kinked wall stays pinned in the same place with a reduced wall thickness (see frame II in Fig. 6(d)). Therefore, when the transverse field is removed (see frames III), the system goes back to its initial magnetization state for $\mu_{0} H_{\mathrm{x}}$ $=-8 \mathrm{mT}$ whereas for $\mu_{0} H_{\mathrm{x}}=8 \mathrm{mT}$ it has reached a new state with a pinned flat wall. Thus, only in this case, there is a net $\Delta M_{\mathrm{y}}=-0.19 M_{\mathrm{S}}$ between the initial and final magnetization states caused by the net backward motion of the pinned wall. 
The sign dependence of the calculated rectification is directly connected to the chirality of the $180^{\circ}$ pinned Neel wall: the transverse field promotes depinning of the kinked wall only when it is applied parallel to the magnetization within the wall core. This is because the influence of a transverse field on a Neel wall can be two fold: in the first place, a hard axis field reduces the overall DW angle and, correspondingly, the total DW energy mainly due to the smaller magnetostatic energy stored in the Neel wall tails. This is an even effect that would decrease the line tension associated to the kinked wall similarly for positive and negative
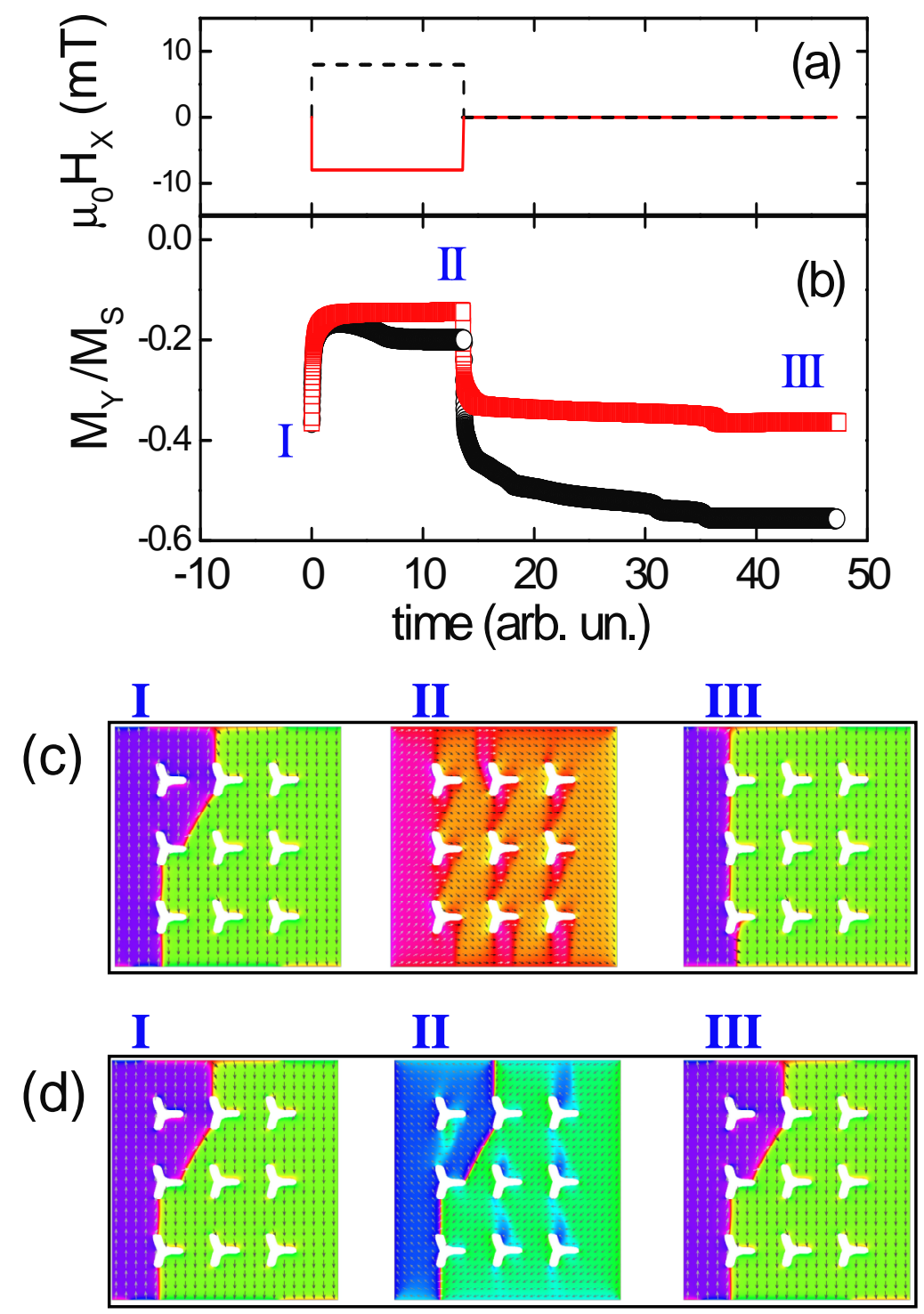

Figure 6 (colour online) (a) Transverse field pulses applied to a kinked wall for the micromagnetic simulations; (b) Calculated time dependence of the easy axis magnetization under a pulse of transverse field: $\mathrm{M}_{\mathrm{y}}(\mathrm{t})$ for positive field pulse is indicated by circles and $\mathrm{M}_{\mathrm{y}}(\mathrm{t})$ for negative field pulse is indicated by squares. (c) Simulated magnetization configurations at (I) initial stage, (II) relaxed under transverse field $\mu_{0} H_{\mathrm{x}}=8 \mathrm{mT}$ and (III) remanence. (d) Simulated magnetization configurations at (I) initial stage, (II) relaxed under transverse field $\mu_{0} H_{\mathrm{x}}=-8 \mathrm{mT}$ and (III) remanence. Note the differences in domain wall width in frames II for positive/negative transverse field. 
transverse fields. In the second place, the energy of the Neel wall core is quite different depending on its orientation relative to the hard axis field. This results in a significant broadening of the Neel core for positive fields only (see frames II in Fig. 6) so that pinning by the patterned holes would be weakened by finite size effects [11-13]. This could allow upward kink propagation (which is the softer propagation mode of the system) driven by DW line tension in the presence of pinning centers of strongly reduced efficiency. For a Neel wall of opposite chirality the transverse field kink depinning would take place at negative fields but would again result in upward kink motion with the same sign of $\Delta M_{\mathrm{y}}$ given by the crossed ratchet potential. It is interesting to note that the sensitivity to the sign of the hard axis field is only experimentally found in the ac transverse accommodation experiments (Fig. 5) in which the same pinned DW is observed during the whole measuring sequence. However, for the dc experiments shown in Fig. 4, in which a constant transverse field is present both during DW nucleation and propagation the observed behavior is even in $H_{\mathrm{x}}$. This can be attributed to a preferred DW nucleation with a chirality such that core orientation is along the applied transverse field, i.e. opposite for positive/negative fields [21,22].

\section{Conclusion}

In summary, the asymmetric DW propagation that gives rise to crossed ratchet behavior has been studied by micromagnetic simulations in square arrays of asymmetric holes with two different shapes: triangle and arrow. Triangle holes result in a stronger forward/backward asymmetry $\Delta \mathrm{H}_{\mathrm{F}} / \Delta \mathrm{H}_{\mathrm{B}}$ for flat wall propagation due to the smaller $\Delta \mathrm{H}_{\mathrm{F}}$ in comparison with arrow holes. However, arrow holes are more suitable for the observation of crossed ratchet effects since they present a larger available field range $\mu_{0} \Delta \mathrm{H}_{\text {Cross }}=\mu_{0} \mathrm{H}_{\mathrm{F}}-\mu_{0} \mathrm{H}_{\mathrm{U}}$ in which the only DW propagation modes available correspond to kink motion. The simulated values $\mu_{0} \Delta \mathrm{H}_{\text {Cross }}$ of the order of $0.1 \mathrm{mT}$ are in good agreement with experimental observations.

Transverse field experiments have shown that domain wall propagation across the array of asymmetric defects is significantly affected by the presence of a hard axis field component: first, dc transverse fields of enough amplitude enhance the easy axis asymmetric accommodation and, also, ac transverse fields are found to be rectified by the crossed ratchet potential. These results can be attributed to the reduction in DW energy by the hard axis field and the polarity dependent broadening of the Neel wall core.

\section{Acknowledgements}

Work supported by Spanish MICINN (FIS2008-06249) and by Principado de Asturias FICYT (PCTI IB08-106). 


\section{References}

[1] Allwood DA, Xiong G, Faulkner CC, Atkinson D, Petit D and Cowburn RP 2005 Science 3091688

[2] Parkin SSP, Hayashi M and Thomas L 2008 Science 320190

[3] Allwood D, Xiong G and Cowburn RP 2004 Appl. Phys. Lett. 852849

[4] Faulkner CC, Allwood DA, and Cowburn RP 2008 J. Appl. Phys. 103073914

[5] Himeno A, Okuno T, Kasai S, Ono T, Nasu S, Mibu K, and Shinjo T 2005 J. Appl. Phys. 97066101

[6] Savel'ev S, Rakhmanov A and Nori F 2005 New J. Phys. 782

[7] Piao H-G, Lee H, Yoon J, Kim D-H, You C-Y and Kim TW 2010 IEEE Trans. Magn. 461844

[8] Yamaguchi A, Kishimoto T, and Miyajima H 2010 Appl. Phys. Express 3093004

[9] Pérez-Junquera A, Marconi VI, Kolton AB, Alvarez-Prado LM, Souche Y, Alija A, Velez M, Anguita JV, Alameda JM, Martin JI, and Parrondo JMR 2008 Phys. Rev. Lett. 100037203.

[10] Hayashi M, Thomas L, Rettner C, Moriya R, Jiang X, and Parkin SSP 2006 Phys. Rev. Lett. 97 207205

[11]Marconi VI, Kolton AB, Capitán JA, Cuesta JA, Pérez-Junquera A, Vélez M, Martín JI and Parrondo JMR 2011 Phys. Rev. B $\mathbf{8 3} 214403$

[12] Alija A, Perez-Junquera A, Rodriguez-Rodriguez G, Velez M, Marconi V I, Kolton A B, Anguita J V, Alameda J M, Parrondo J M R and Martin J I 2009 J. Phys. D: Appl. Phys. 42045001

[13]Pérez-Junquera A, Rodríguez-Rodríguez G, Vélez M, Martín JI, Rubio H and Alameda JM $2006 \mathrm{~J}$. Appl. Phys. 99033902

[14]Pérez-Junquera A, Martín JI, Vélez M, Alameda JM, Anguita JV, Briones F, González EM and Vicent JL 2004 Nanotechnology 15 S131

[15]Morales R, Martín JI, Vélez M and Alameda JM 2004 Eur. Phys. J. B 40463

[16]Donahue MJ and Porter DG, OOMMF User's Guide, Version 1.0, Interagency Report NISTIR 6376. National Institute of Standards and Technology, Gaithersburg, MD, Sept. 1999; http://math.nist.gov/oommf.

[17]Hubert A and Schäfer R, Magnetic Domains, (Springer-Verlag, Berlin, 1998).

[18]Bryan MT, Schrefl T, Atkinson D and Allwood DA 2008 J. Appl. Phys. 103073906

[19]Glathe S, Berkov I, Mikolajick T, and Mattheis R 2008 Appl. Phys. Lett. 93162505

[20]Chikazumi S, Physics of Magnetism (Krieger, Malabar, 1978) p. 314

[21]Eastwood DS, Bogart LK and Atkinson D 2010 Acta Physica Polonica A 118 719;

[22]Eastwood DS, King JA, Bogart LK, Cramman H, and Atkinson D 2011 J. Appl. Phys. 109013903 